\title{
Cervical cancer screening in low-income countries: a report on the implementation of cervical screening in Luzon, Philippines
}

\author{
Samantha Carr', Charlotte Hespe ${ }^{1}$ \\ 1 School of Medicine, University of Notre Dame, Australia \\ Keywords: cancer screening, philippines, cervical cancer \\ https://doi.org/10.29392/001c.14149
}

\section{Journal of Global Health Reports}

Vol. 4, 2020

\begin{abstract}
Background
Cervical cancer is the second leading cause of cancer death among women in the Philippines. This study is the first clinical audit of a cervical cancer screening program in a socioeconomically deprived community in rural Philippines. The aim of this study was to quantify the proportion of women screened who were identified as having no cervical abnormality, low-grade squamous intraepithelial lesion (LSIL/CIN 1), high grade squamous intraepithelial lesion (HSIL/CIN 2) or carcinoma in situ (CIS) and to measure the proportion of women who received appropriate follow-up.
\end{abstract}

\section{Methods}

Retrospective audit of data from medical records collected between 2013-2017 in Santo Tomas, Luzon, Philippines.

\section{Results}

625 women presented for screening and 615 satisfactory screening tests were conducted. $88.4 \%$ of women had a normal result (95\% confidence interval, $\mathrm{CI}=85.8-90.9), 10.2 \%$ had low-grade changes (95\% CI=7.8-12.6), $0.3 \%$ had high-grade changes (95\% CI=0.0-1.0) and $0.5 \%$ were diagnosed with cervical cancer $(95 \% \mathrm{CI}=0.0-1.0)$. Follow-up was poor for women with normal (26.1\%; 95\% CI=21.6-31.1) and abnormal (27.1\%; 95\% CI=15.3-41.8) results.

\section{Conclusions}

Our experience confirms previous studies surrounding barriers to follow up and treatment within developing countries and should be complemented with qualitative research to further explore these barriers. Given low rates of follow-up, a screen-and-treat approach and/or human papilloma virus (HPV) vaccination may be more appropriate within this community.

Cervical cancer is the second most common cancer among women in the Philippines, accounting for $14 \%$ of female cancers, and is the second leading cause of cancer mortality among Filipino women, accounting for $6 \%$ of female cancer deaths. ${ }^{1}$ The 5 -year survival rate for cervical cancer remained unchanged at $44 \%$ between 1980 and $2010 .^{2}$ This has been attributed to late-stage diagnosis in the majority of patients and unaffordable or inaccessible treatment. ${ }^{3}$

Risk factors for cervical cancer are more prevalent among Filipino women than the global community and include young age at first intercourse, low socioeconomic status, high parity, smoking, use of oral contraception and risky sexual behaviours. ${ }^{3}$

Currently, there is no national cervical cancer screening program in the Philippines and cervical cancer screening coverage was estimated to be less than $8 \%$ nationwide in 2003 , and only $5 \%$ in rural areas. ${ }^{4}$ Several studies have sought to determine the most effective method for screening and prevention in an economically developing setting including cytology based methods (e.g. pap smear), human papilloma virus, HPV-DNA testing, visual inspection methods and HPV vaccination. The main barrier to cytologybased screening in economically deprived countries is the lack of pathology services, infrastructure or trained practitioners. ${ }^{5}$

A cost-utility analysis of different methods for cervical cancer screening and prevention in the Philippines found that screening women aged 35-45 every 5 years using visual inspection with acetic acid (VIA) was the most cost-effective strategy, whilst HPV vaccination of girls at age 11 would also be cost-effective if there was high coverage (>80\%) and providing the vaccine resulted in lifelong immunity. ${ }^{2}$ The Philippine Department of Health (DOH) currently recommends that cervical cancer screening targets women aged 25-55 years every 5-7 years using the VIA method. ${ }^{2}$ 
This study is the first clinical audit of a cervical cancer screening program that was implemented in a socioeconomically deprived community in rural Philippines from November 2013. The program uses visual inspection with acetic acid (VIA) and visual inspection with Lugol's iodine (VILI) and targets women aged 18-70 years old.

The aim of this study was to assess the effectiveness of a cervical cancer screening program in the community of Santo Tomas, Luzon, Philippines. We sought to determine the number and demographic characteristics of women in the target population who participated in the cervical cancer screening program between November 2013 and November 2017 in Santo Tomas, Luzon, Philippines, and the proportion of these participants who had never been screened before, to quantify the proportion of women screened who were identified as having no cervical abnormality, LSIL/CIN 1, HSIL/CIN 2 or CIS and to measure the proportion of women who had a normal versus abnormal who received appropriate follow-up, rescreening or review according to treatment protocol.

\section{METHODS}

\section{BACKGROUND TO THE TEAM PHILIPPINES PROJECT}

This study is a retrospective audit of data from medical records collected during 9 separate weeks of women's health clinics run in July and November from 2013-2017 in the public housing community of Santo Tomas, Laguna, Luzon, Philippines. The women's health clinic forms part of a larger project Team Philippines implemented since 2013 to provide primary and preventative medical and dental care, community projects and infrastructure.

The residents of this public housing community previously lived in makeshift accommodation alongside the waterways of Manila and were displaced by major floods in 2009. They were re-housed by the government to the region of Laguna, roughly $80 \mathrm{~km}$ southeast of Manila. The closest basic healthcare facilities are located in Calauan, $5 \mathrm{~km}$ away, although for most health care services patients are required to travel $80 \mathrm{~km}$ to Manila.

The local clinics in Calauan do not have access to pathology or imaging services and must refer patients on if these services are required. Prior to 2013 when Team Philippines initiated the cervical screening program, there were no screening clinics available in the region. The Regional Health Unit in Calauan has recently initiated a weekly cervical cancer screening clinic using the VIA method independent of the Team Philippines women's health clinic. There are no pathology, imaging or treatment services offered at this clinic and any patients with abnormal findings are referred to Manila.

The Team Philippines women's clinic is run in a designated room within the Santo Tomas housing estate's community centre over 6 days twice a year. Initially women presented spontaneously directly to the women's clinic, however from November 2015 patients have been triaged and seen on a "first come, first served" basis. From July 2017, patients who had a previous abnormal result were identified the week prior to clinic and asked to return by the local community health nurse.
Upon arrival at the women's clinic, a brief medical and sexual history is taken by a medical student, who is supervised by a senior clinician. History taking is assisted by a local interpreter and details of the history are entered on a hard-copy "colposcopy" template form (see Online Supplementary Document). There is access to a computer with Medical Director ${ }^{\circledR}$ database to review patient files and previous screening results including demographic information, religion, ethnicity, general medical history, sexual history, obstetric history, smoking and alcohol status, vital observations and examination findings. The screening procedure is explained to the patient through the interpreter and the patient is consented. Patients are offered a chaperone.

Patients are then led to a private examination space behind a curtain and are provided with a modesty sheet. Prior to November 2016, the women's clinic was run in a smaller room without electricity and the procedure was performed with head torches. From November 2016, the clinic has been moved to a larger room with electricity and the procedure is conducted with both overhead lighting and head torches. Medical students either observe the procedure or are supervised by a senior doctor. A bimanual examination is performed, followed by speculum examination.

The current cervical screening program uses the visual inspection method to detect abnormal lesions, which involves placing $4 \%$ diluted acetic acid onto the squamocolumnar junction (SCJ) of the external os of the cervix for 1 minute and examining for aceto-white changes (VIA method). The senior doctor is equipped with a magnifying head torch. If changes are detected, the same method can be repeated with Lugol's iodine to look for unchanged yellow areas (VILI method).

Results are recorded on the colposcopy template forms including diagrammatic representation of the visual inspection and identification into categories Normal, LSIL/CIN 1, HSIL/CIN 2 or CIS (see Online Supplementary Document). Details of the consultation are also recorded in the Medical Director $^{\circledR}$ database. The computer used in the women's clinic is connected via server to the other computers being used in other clinics at the time, allowing patients to flow through multiple clinics throughout the day and have their data recorded and accessed. The double recording procedure on hard-copy template and electronic version was implemented to safeguard against technical difficulties with the server. The paper forms are scanned following each trip and uploaded onto each patient's Medical Director ${ }^{\circledR}$ file.

Patients with a normal result are asked to return in 2 years, patients with low-grade changes are asked to return in 6 months, patients with high-grade changes are referred to a healthcare clinic Manila for biopsy and further investigation and patients with suspected cervical cancer are referred to the Philippine General Hospital in Manila for evaluation and treatment. Each consultation lasts roughly 20 minutes and patients are given the opportunity to ask questions. This protocol differs from the Philippine Department of Health recommendations for cervical screening which recommends screening using the visual inspection (VIA) method for women aged 35-55 years every 5-7 years. The current protocol follows the previous Australian guidelines for Pap smear screening, which was 2-yearly for women aged $18-70 .^{6}$ 
From November 2017, patients have been given an information pamphlet to read whilst waiting to be seen which details the importance of cervical cancer screening and outlines the procedure.

\section{SAMPLE GROUP ELIGIBILITY CRITERIA}

Patients who were eligible to participate in the program were women aged 16-72 who had a cervix. If they were menstruating at the time of the clinic, they were advised to return at the next opportunity. Women who had undergone hysterectomy were offered vaginal examination however the VIA/VILI method cannot be performed on these women.

Some patients returned for follow-up before the recommended time period. Some of these patients were rescreened whilst others were asked to return in the recommended time frame. Patients who participated all gave informed consent.

\section{DATA COLLECTION}

Paper records from each clinic were collated onto a de-identified Excel spread sheet including patient age, smoking status, body mass index, gravidity and parity, previous screening results and contraception use, as well as the results from each visual inspection examination. This data was supplemented with electronic data recorded at the time of visit using the Medical Director ${ }^{\circledR}$ database. Smoking status was considered positive if the patient had ever smoked and contraceptive use was considered positive if use was current at time of interview. Women were considered at risk of unintended pregnancy if they were still menstruating.

\section{DATA ANALYSIS}

De-identified demographic and clinical data were exported from the Excel spread sheet into SPSS for analysis. Demographic data, sexual history and baseline screening results were reported using frequencies and $95 \%$ confidence intervals. T-test and Chi-square test were used to compare appropriate follow-up. Significance was set at $P \leqslant 0.05$.

\section{RESULTS}

A total of 624 women aged 16-72 presented to the women's clinic for cervical cancer screening over 9 weeks of clinic, resulting in 764 visits recorded. Of these women, 5 were excluded because they did not meet the eligibility criteria: 2 were menstruating at the time of screening and did not have an opportunity to return to a subsequent clinic during the study period and 3 had undergone hysterectomy and VIA/ VILI was not performed.

Of the 619 women who were eligible for screening, 615 had a satisfactory screening test performed. The clinician was unable to locate the cervix or visualise the squamocolumnar junction in three women and one woman had undiluted acetic acid applied to her cervix unintentionally resulting in inability to interpret her result.

Demographic characteristics, sexual history and results of baseline screen are reported in Table 1 ( $A$ and $B$ ).
Results of screening are shown in Table 2. Over the course of the study, $11.0 \%$ of women had an abnormal result. Three women were diagnosed with cervical cancer (see Case Studies for further information).

\section{FOLLOW-UP}

Table 3 outlines the proportion of patients who returned following normal and abnormal first screen.

Upon initial analysis, there appeared to be statistically significant differences between return rates in the normal and abnormal groups (Chi-square, $\chi^{2}(4, \mathrm{n}=619)=33.53$, $P \leqslant 0.005)$. However, this is confounded by a large proportion of patients for whom the screening interval period had not yet elapsed, highlighted in Table 4 .

There was no significant difference in follow-up rates between those with normal versus abnormal results when analysing only patients for whom the screening period had elapsed $\left(\chi^{2}(1, \mathrm{n}=400)=0.02, P=0.89\right)$.

\section{CASE STUDIES OF WOMEN WITH CERVICAL CANCER DIAGNOSED DURING SCREENING PROGRAM}

Patient 1 was diagnosed with cervical cancer on clinical examination in July 2014 at age 67. Her previous screening test was in 1992 and she stated it was normal. She had been menopausal since 1992, had never smoked and had 3 children. During her examination in July 2014, a hard mass was palpable on internal examination and a bleeding tumour was visualized at the apex of the vagina. She was referred to Philippine General hospital in Manila where cervical cancer was confirmed as a low-grade tumour. Histopathology results were not made available to the referring doctor. The tumour was too advanced for surgery however she was treated with radiation and chemotherapy until January 2015. She returned to our clinic in November 2014, July 2015 and July 2017 for follow-up where her examination findings were stable and reported as significant scarring from radiotherapy. She has been encouraged to return to our clinic every 2 years or earlier if symptoms develop.

Patient 2 was diagnosed with cervical cancer in November 2016 at age 44 . She presented to our clinic following what she thought was an abnormal screening test at a local clinic in July 2016, requesting a second opinion. She reported 1 year of post-coital bleeding and 2 years of white vaginal discharge. She was pre-menopausal with 5 children. She did not use contraception and her smoking status was unknown. On bimanual examination, there was a large amount of watery discharge. Speculum examination revealed a cervical tumour that was ulcerating and friable. The lesion bled copiously, appeared highly vascularized and was visually consistent with cervical carcinoma. She was referred to Philippine General Hospital in Manila where she underwent a total hysterectomy and was offered adjuvant radiotherapy. After commencing radiotherapy, the patient discontinued treatment stating that radiotherapy was too expensive and difficult to access. She returned to work. We were unable to re-engage with her during the study period.

Patient 3 was diagnosed with cervical cancer in November 2017 at age 37. She presented to our clinic for first-time screening. She reported a change in her menstrual pattern 
Table 1. Demographic characteristics, sexual history and baseline screening results $(n=619)$

\begin{tabular}{|c|c|}
\hline A. Demographic characteristic & Mean (SD) \\
\hline Age & $37.6(+/-11.5)$ \\
\hline Body mass index & $23.7(+/-4.2)$ \\
\hline Gravidity & $3.4(+/-2.4)$ \\
\hline Parity & $3.1(+/-2.2)$ \\
\hline B. Baseline screening result & $\mathrm{n}(\% ; 95 \% \mathrm{Cl})$ \\
\hline \multicolumn{2}{|l|}{ Ever smoked $(n=619)$} \\
\hline Yes & $73(11.8 ; 9.3-14.3)$ \\
\hline No & $519(83.8 ; 80.9-86.7)$ \\
\hline Unknown & $27(4.4 ; 2.8-6.0)$ \\
\hline \multicolumn{2}{|c|}{ Current contraception use, at risk of unintended pregnancy $(n=520)$} \\
\hline Yes & $239(46.0 ; 41.7-50.2)$ \\
\hline No & $281(54.0 ; 49.8-58.3)$ \\
\hline \multicolumn{2}{|c|}{ Contraception method, at risk of unintended pregnancy $(n=520)$} \\
\hline Oral contraceptive pill & $147(28.3 ; 24.4-32.1)$ \\
\hline Tubal ligation & $38(7.3 ; 5.1-9.5)$ \\
\hline IUD & $22(4.3 ; 2.5-6.0)$ \\
\hline Implanon & $17(3.3 ; 1.7-4.8)$ \\
\hline Depot injection & $8(1.5 ; 0.5-2.6)$ \\
\hline Condoms & $7(1.3 ; 0.4-2.3)$ \\
\hline None & $281(54 ; 49.8-58.3)$ \\
\hline \multicolumn{2}{|l|}{ Previous known STI (n=619) } \\
\hline Yes & $4(0.6 ; 0-1.3)$ \\
\hline No & $615(99.4 ; 98.7-100)$ \\
\hline \multicolumn{2}{|l|}{ Previous vaginal infection $(n=619)$} \\
\hline Yes & $3(0.5 ; 0.0-1.0)$ \\
\hline No & $616(99.5 ; 99.0-100)$ \\
\hline \multicolumn{2}{|l|}{ Previous cervical procedure $(n=619)$} \\
\hline Cryotherapy & $0(0.00)$ \\
\hline Cone biopsy & $0(0.00)$ \\
\hline LEEP & $0(0.00)$ \\
\hline \multicolumn{2}{|c|}{ Previous cervical cancer screen $(n=619)$} \\
\hline Yes & $199(32.1 ; 28.5-35.8)$ \\
\hline No & $416(67.2 ; 63.5-70.9)$ \\
\hline Unknown & $4(0.6 ; 0.0-1.3)$ \\
\hline \multicolumn{2}{|l|}{ Result of previous screen ( $n=199)$} \\
\hline Normal & $74(37.2 ; 30.5-43.9)$ \\
\hline Low grade changes/CIN 1 & $6(3.0 ; 0.6-5.4)$ \\
\hline High grade changes/CIN 2/3 & $0(0.00)$ \\
\hline Cancer & $0(0.00)$ \\
\hline Infection & $7(3.5 ; 1.0-6.1)$ \\
\hline Unknown & $112(56.3 ; 49.4-63.2)$ \\
\hline \multicolumn{2}{|c|}{ Cervical screen result at baseline $(n=619)$} \\
\hline Normal & $559(90.3 ; 88.0-92.6)$ \\
\hline Low grade changes/CIN 1 & $52(8.4 ; 6.2-10.6)$ \\
\hline High grade changes/CIN 2/3 & $1(0.2 ; 0.0-0.5)$ \\
\hline Cancer & $3(0.5 ; 0.0-1.0)$ \\
\hline
\end{tabular}




\begin{tabular}{ll}
\hline A. Demographic characteristic & Mean (SD) \\
\hline \multicolumn{1}{c}{ Unsatisfactory screen } & $4(0.6 ; 0.0-1.3)$ \\
\hline CI - Confidence Interval, CIN - Cervical Intraepithelial Neoplasia, IUD - Intrauterine Device, LEEP- Loop Electrosurgical Excision Procedure, STI - Sexually Transmitted Infection. \\
\hline \\
Table 2. Number of women with abnormal screening result during study period $(\mathrm{n}=619)$ \\
\hline Cervical screen result & Number of women $(\% ; 95 \% \mathrm{CI})$ \\
\hline Normal & $551(89.0 ; 85.8-90.9)$ \\
LSIL/CIN 1 & $63(10.2 ; 7.8-12.6)$ \\
HSIL/CIN 2 & $2(0.3 ; 0.0-0.8)$ \\
Cancer & $3(0.5 ; 0.0-1.0)$ \\
Total & $619(100)$ \\
\hline
\end{tabular}

CI - Confidence Interval, CIN - Cervical Intraepithelial Neoplasia, HSIL - High-grade Squamous Intraepithelial Lesion, LSIL - Low-grade Squamous Intraepithelial Lesion, SD - Standard Deviation.

Table 3. Follow-up of patients after first visit $(n=619)$

\begin{tabular}{lll}
\hline & Normal result $(\% ; 95 \% \mathrm{Cl})$ & Abnormal result $(\% ; 95 \% \mathrm{Cl})$ \\
Never returned & $260(46.2 ; 42.0-50.4)$ & $35(62.5 ; 48.5-75.1)$ \\
\hline Returned on time & $14(2.5 ; 1.4-4.1)$ & $6(10.7 ; 4.0-21.9)$ \\
Returned early & $54(9.6 ; 7.3-12.3)$ & - \\
\hline Returned late & $24(4.3 ; 2.7-6.3)$ & $7(12.5 ; 5.2-24.1)$ \\
Screening interval not elapsed by end of study period & $211(37.5 ; 33.5-41.6)$ & $8(14.3 ; 6.4-26.2)$ \\
Total & 563 & 56 \\
\hline
\end{tabular}

CI - Confidence Interval.

Table 4. Follow-up of patients after first visit with completed screening period

\begin{tabular}{lll}
\hline & Normal result (\%; 95\% Cl) & Abnormal result (\%; 95\% Cl) \\
\hline Never returned & $260(73.9 ; 68.9-78.4)$ & $35(72.9 ; 58.2-84.7)$ \\
Returned & $92(26.1 ; 21.6-31.1)$ & $13(27.1 ; 15.3-41.8)$ \\
Total & 352 & 48 \\
\hline
\end{tabular}

CI - Confidence Interval.

with menorrhagia every 6 weeks for a year. She denied inter-menstrual or post-coital bleeding. She was a non-smoker, did not use contraception and had two children. On bimanual examination there was a polypoid tumour involving the cervix, with no uterine enlargement or adnexal tenderness. On speculum examination there was a $5 \mathrm{~mm}$ lesion at 12 o'clock that was friable and had a cauliflower-like appearance. The lesion was VIA/VILI positive. She was referred to Manila for gynaecological assessment, colposcopy and biopsy where her initial assessment was thought to be a high-grade lesion rather than cervical cancer. At the time of writing she is still undergoing assessment and treatment. She was asked to return to see us for follow-up in July 2018.

\section{DISCUSSION}

Herein we report the first clinical audit of a cervical cancer screening program implemented in the rural, socio-economically deprived community of Santo Tomas, Laguna, Luzon, Philippines. We found that 68 women (11\% of those screened) had an abnormal result, including 3 with cervical cancer. There was no statistically significant difference between follow up rates for women with normal versus abnormal results after accounting for those for whom the screening period at not yet elapsed.

The Philippines has no national cervical cancer screening register or program or national HPV vaccination pro- 
gram. This is compounded by a lack of access to local clinics, poor health literacy and high prevalence of risk factors for cervical cancer. With the addition of low resources in a rural setting, the population of Santo Tomas forms one of the most vulnerable in the world. The Team Philippines projected has therefore been implemented to combat these inequalities to reduce the morbidity and mortality associated with cervical cancer.

Over the five-year study period, 68 of 615 women (11\%) had an abnormal screening result, including 63 (10.2\%) with LSIL/CIN 1, 2 (0.3\%) with HSIL/CIN 2 and 3 (0.5\%) with cervical cancer. These findings reflect a similar prevalence of low-grade cervical lesions and cervical cancer as other studies in developing countries around the world. 7,8

The majority of patients (67\%) had never had a cervical cancer screening test before presentation to our clinic. Of those that had previously been screened, the majority (56\%) did not know the result of their screening test with only $3 \%$ reporting a previous abnormal result. No patients reported having previous cervical procedures conducted including cryotherapy, cone biopsy or loop electrosurgical excision procedure (LEEP) procedures. These results suggest a lack of health literacy surrounding the nature of cervical cancer screening as well as potential miscommunication between clinician and patient using a local interpreter. The closest health clinic to the community also conducts cervical cancer screening however they do not perform cryotherapy and patients must be referred to Manila for further treatment or investigation. Therefore it is possible that no patient has ever undergone a therapeutic cervical procedure.

The women of Santo Tomas are at increased risk of cervical cancer due to high prevalence of risk factors and poor health literacy, particularly with respect to women's hygiene, fertility, contraception and sexually transmitted infections. Many women had never had cervical cancer screening prior to being seen in our clinic, and, reflecting their lack of knowledge, some believed the purpose of the screening procedure was to clean the cervix in preparation for pregnancy. Despite many women having contact with the health care system for antenatal visits and birthing, the women do not appear to have access to education regarding basic sexual health.

Women in the community of Santo Tomas have a higher use of contraception compared to the national average. Our results showed $46 \%$ of women use modern methods of contraception (e.g. sterilization, intrauterine contraceptive device, oral contraceptive pill, implants, condoms etc.). This is compared to the national average of currently married women of $40 \%$ (42\% in rural areas and $38 \%$ in urban areas). ${ }^{9}$ However, despite higher rates of contraception use, women in Santo Tomas have a high fertility rate of 3.1 live births/ woman compared to the national total fertility rate of 2.7 live births/woman in 2017. ${ }^{9}$ This figure remains high when compared to rural women overall where the total fertility rate is 2.9 live births/woman compared to the urban total fertility rate of 2.4 live births/woman. ${ }^{9}$ This paradoxical finding may suggest a lack of health education surrounding fertility and correct contraception use or may be attributed to intermittent use of contraception due to insufficient income to afford contraception. In July 2017, we began running health education sessions focussing on fertility and contraception that have been well attended by over 400 women.

We found that $11.8 \%$ of women had ever smoked. This is compared to the national average of $4.9 \%$ of women in 2015 who were current cigarette smokers, smoking an average of 8.6 cigarettes per day. ${ }^{10}$ This study did not include data on the average number of cigarettes smoked however it is assumed to be low given anecdotal evidence that many women cannot afford to smoke more than 2-3 cigarettes per day. Finally, we had very few reports of previous sexually transmitted infection (STI). We propose this result to be due to a lack of pathology facilities in the community as well as poor health literacy surrounding signs and symptoms of STIs.

Our experience confirms previous studies surrounding barriers to follow up and treatment within developing countries. ${ }^{5,11}$ Follow-up was poor for women with normal (26.1\%; 95\% CI=21.6-31.1) and abnormal (27.1\%; 95\% $\mathrm{CI}=15.3-41.8)$ results. This may reflect low health literacy surrounding the importance of follow-up, poor communication via local health interpreters, competing health priorities and stigma associated with a diagnosis of a cervical abnormality. To address this issue, from July 2017, we sought to actively recall patients via the local community health nurse and attempted to educate women on the benefits of cervical cancer screening through circulating information pamphlets. This is an ongoing screening program and future studies will aim to complete follow-up data for patients with an incomplete screening interval and allow a more complete comparison between follow-up rates amongst women with normal versus abnormal screening results.

In order to improve outcomes, qualitative research should be undertaken to better understand the barriers to follow-up. Alternative approaches to cervical cancer screening that require less follow-up should also be considered. Goldie et al. ${ }^{12}$ found that the most cost-effective strategies for cervical cancer prevention in developing countries were those requiring fewer visits as this increased patient return for follow-up and treatment. They found a $40 \%$ lifetime reduction in cervical cancer by screening twice, using either VIA or HPV testing, at ages 35 and $40 .^{12}$ The World Health Organisation and International Agency for Research on Cancer $^{8}$ support a screen-and-treat approach in low resource settings where women are treated with cryotherapy at the same visit if a low-grade lesion is detected. A study conducted across six African countries found that only $50 \%$ of women treated with cryotherapy returned for follow-up however all of them had a subsequent negative VIA test. ${ }^{8}$ Finally, a cost-utility analysis in the Philippines found that HPV vaccination of girls at age 11 would be cost-effective if coverage was greater than $80 \%{ }^{2}$ Further investigation into the practicality of implementing such a vaccination program would be beneficial.

There were several limitations to the study. Firstly, data from the triage station suggests that up to $30 \%$ of patients who presented to triage requesting a ticket for the women's health clinic were not seen in the women's clinic. The reasons for this attrition is unknown, however possible reasons include long waiting times, other clinics having higher priority for the patient and inadequate space in the women's clinic. This attrition may have resulted in symptomatic 
women not being screened due to this lack of resources. Given there is no national register for cervical cancer screening in the Philippines, we do not have access to the registers of other health care services and are unable to confirm if patients have been screened and followed up elsewhere.

Secondly, we are unable to confirm the sensitivity and specificity of the VIA method within this clinic without a gold standard comparison. All interpretations were carried out by trained clinicians, with the majority reported by a single clinician. A 2011 meta-analysis reported a sensitivity and specificity for VIA of $80 \%$ (range $79-82 \%$ ) and $92 \%$ (range 91-92\%) respectively. ${ }^{13}$ Further studies using HPVDNA testing could confirm the sensitivity and specificity of VIA performed within the current context.

Thirdly, the data collection process was somewhat inconsistent and several entries were missing, particularly for body mass index and smoking status. Only 3 patients reported ever having a sexually transmitted infection and no patients reported a history of HIV or HPV. This finding may be due to lack of access to pathology services, or may be a function of the stigma associated with sexually transmitted infections and the fact that the consultation involved an interpreter from the local community. Several studies have found that the use of inexperienced interpreters in healthcare settings and healthcare research can impact the quality and accuracy of information. ${ }^{14}$ Future clinics could clarify the medical jargon with the local interpreters or engage trained health interpreters.

Finally, there is limited access to demographic information about the community in general. Whilst migration in and out of the community is considered minimal, we are unable to assess what subset of the community are presenting to the clinic and thus are unable to define which characteristics make a woman more or less likely to seek screening.

In conclusion, this study demonstrates a similar prevalence of low-grade cervical lesions and cervical cancer as other studies in developing countries around the world. 7,8 The women of the community of Santo Tomas are at increased risk of cervical cancer given the higher prevalence of risk factors and poor health literacy. Follow-up was poor for women with normal and abnormal results. Future objectives include improving the screening questionnaire template to gain a more accurate risk profile, improving patient and interpreter health literacy through education sessions, qualitative research to better understand the barriers to follow-up and considering alternative methods for cervical cancer prevention including screen-and-treat approaches, HPV-DNA testing or HPV vaccination.
Acknowledgements: We would like to thank Dr Zelda Doyle (University of Notre Dame Australia) for her assistance with the statistical analysis.

Disclaimers: Views expressed in the below manuscript are those of the authors and do not necessarily reflect those of the University of Notre Dame Australia or funders

Statement of compliance with NHMRC guidelines for ethical research: This applied research project report complies with the Australian National Health and Medical Research Council (NHMRC) National Statement on Ethical Conduct in Human Research. A Human Ethics Research Committee (HREC) low-risk ethics approval from the University of Notre Dame Australia was obtained as the project was a clinical audit of medical records and carried a low risk as per paragraphs 2.1.6 and 2.1.7 of the National Statement (HREC reference: 016204S. Approval date: 14/12/2016. Amendment date: 03/02/2020). There was no participant involvement required in the research and all data was non-identifiable.

Funding: The project described in the below manuscript forms part of a larger project named Team Philippines, a not-for-profit organisation that runs health, dental, optometry, women's health, infrastructure, education and livelihood programs within the community of Calauan, Luzon, Philippines. This project is invested in the education and training of medical students from the University of Notre Dame Australia in the Asia-Pacific region and thus has received funding from the Australian Government Department of Foreign Affairs and Trade through the New Colombo Plan, Asia Postgraduate scholarships and Asia-bound scholarships.

Authorship contributions: SC and $\mathrm{CH}$ contributed to the study design, draft and final version of the manuscript.

Competing interests: The authors declare no affiliation with or involvement in any organisation or entity with any financial interest or non-financial interest in the subject matter or materials discussed in this manuscript.

\section{Correspondence to:}

Dr Samantha Carr, MD

School of Medicine, University of Notre Dame Australia

Samantha.carr@live.com

Associate Professor Charlotte Hespe, MBBS (Hons), FRACGP, FAICD

School of Medicine, University of Notre Dame Australia Charlotte.hespe@nd.edu.au 


\section{REFERENCES}

1. Bruni L, Barrionuevo-Rosas L, Albero G, Aldea M, Serrano B, Valencia S, et al. Human Papillomavirus and Related Diseases Report: Philippines. ICO Information Centre on HPV and Cancer. 2016.

2. Guerrero AM, Genuino AJ, Santillan M, et al. A cost-utility analysis of cervical cancer screening and human papillomavirus vaccination in the Philippines. BMC Public Health. 2015;15(750). doi:10.1186/s1288 9-015-2046-1

3. Domingo EJ, Dy Echo AVV. Epidemiology, prevention and treatment of cervical cancer in the Philippines. Journal of Gynecologic Oncology. 2009;20(1):11-16. doi:10.3802/igo.2009.20.1.11

4. World Health Organization. World Health Survey Results: Report of Philippines. Geneva: World Health Organization; 2003.

5. Catarino R, Petignat P, Dongui G, Vassilakos P. Cervical cancer screening in developing countries at a crossroad: Emerging technologies and policy choices. World Journal of Clinical Oncology. 2015;6(6):281-290. doi:10.5306/wico.v6.i6.281

6. Australian Government, Department of Health. Understanding the National Cervical Screening Program Management Pathway: A Guide for Healthcare Professionals. Australian Government; 2018.

7. Sankaranarayanan R, Nene BM, Shastri SS, Jayant $\mathrm{K}$, Muwonge R, Budukh AM, et al. HPV screening for cervical cancer in rural India. NEJM.

360(14):1385-1394.
8. World Health Organization, International Agency for Research on Cancer. Prevention of Cervical Cancer through Screening Using Visual Inspection with Acetic Acid (VIA) and Treatment with Cryotherapy. A Demonstration Project in Six African Countries: Malawi, Madagascar, Nigeria, Uganda, the United Republic of Tanzania, and Zambia. Geneva, Switzerland: WHO Document Production Services; 2012.

9. Philippine Statistics Authority. Philippines National Demographic and Health Survey 2017: Key Indicators. Quezon City, Philippines, and Rockville, Maryland, USA: PSA and ICF; 2018.

10. Philippine Statistics Authority. Global Adult Tobacco Survey: Executive Summary 2015. Philippines: Quezon City; 2017.

11. Denny L, Quinn M, Sankaranarayanan R. Screening for cervical cancer in developing countries. Vaccine. 2006;24(3):S71-S77.

12. Goldie SJ, Gaffikin L, Goldhaber-Fiebert JD, Gordillo-Tobar A, Levin C, Mahé C, et al. Costeffectiveness of cervical-cancer screening in five developing countries. NEJM. 2005;353(20):2158-2168.

13. Sauvaget C, Fayette JM, Muwonge R, Wesley R, Sankaranarayanan R. Accuracy of visual inspection with acetic acid for cervical cancer screening. International Journal of Gynecology \& Obstetrics. 2011;113(1):14-24.

14. Anazawa R, Ishikawa H, Kiuchi T. The accuracy of medical interpretations: A pilot study of errors in Japanese-English interpreters during a simulated medical scenario. Translation \& Interpreting. 2012;4(1):1-20. 


\section{SUPPLEMENTARY MATERIALS}

\section{Online Supplementary Document}

Download: https://www.joghr.org/article/14149-cervical-cancer-screening-in-low-income-countries-a-report-on-theimplementation-of-cervical-screening-in-luzon-philippines/attachment/38708.docx 